\title{
Improved TOPSIS Model and its Application in the Evaluation of NCAA Basketball Coaches
}

\author{
Qiang Xu ${ }^{1,3}$, Yuan-Biao Zhang ${ }^{2,3}$, Jing Zhang ${ }^{4} \&$ Xin-Guang $\mathrm{Lv}^{2,3}$ \\ ${ }^{1}$ Electrical and Information School, Jinan University, Zhuhai 519070, China \\ ${ }^{2}$ Packaging Engineering Institute, Jinan University, Zhuhai 519070, China \\ ${ }^{3}$ Key Laboratory of Product Packaging and Logistics of Guangdong Higher Education Institutes, Jinan University, \\ Zhuhai 519070, China \\ ${ }^{4}$ School of Humanities, Jinan University, Zhuhai 519070, China \\ Correspondence to: Yuan-Biao Zhang, Packaging Engineering Institute, Jinan University, Zhuhai 519070, China. \\ E-mail: zybt@jnu.edu.cn
}

Received: October 11, 2014 Accepted: October 23, 2014 Online Published: January 28, 2015

doi:10.5539/mas.v9n2p259 URL: http://dx.doi.org/10.5539/mas.v9n2p259

\begin{abstract}
Traditional TOPSIS model has some disadvantages, such as correlations between criteria, uncertainty in obtaining the weights only by objective methods or subjective methods and possibility of alternative closed to ideal point and nadir point concurrently, and many solutions have been proposed regarding these disadvantages. This paper presents a more systematic TOPSIS model, in which the correlations between criteria were overcome by a new method on evaluation index system based on $\mathrm{R}$ cluster analysis. It also proposes a combination weighting method which has considered subjective potency of human and the variance in the data. Besides, the possibility of alternative closed to ideal point and nadir point concurrently was avoided by vertical projection method and the measurement of similarity to solution was simplified by vertical projection distance. The feasibility and validity of this improved TOPSIS model were testified by the evaluation of NCAA basketball coaches after 1939.
\end{abstract}

Keywords: R cluster analysis, combination weighting method, vertical projection method, NCAA basketball coach

\section{Introduction}

TOPSIS (technique for order preference by similarity to solution), firstly presented by Hwang and Yoon, is a simple and efficient multiple criteria method to identify solutions from a finite set of alternatives. However, there are some main disadvantages in traditional TOPSIS model: correlations between criteria, uncertainty in obtaining the weights only by objective methods or subjective methods and possibility of alternative closed to ideal point and nadir point concurrently (Li et al., 2011).

This paper uses $\mathrm{R}$ cluster analysis to overcome the disadvantage - correlations between criteria. In multi-attribute decision making problem, in order not to miss some important criteria, primarily the decision maker would determine as many criteria as possible, which would cause correlations between criteria. Nevertheless, the evaluation system may result in creating too many criteria, which are highly correlated with each other, and can cause inaccuracy in results of the evaluation. Hence, it is necessary to explore the correlations between these variables and classify them into different categories. R cluster analysis, however, can achieve the goals.

Different weighting methods can be used to obtain the weights in TOPSIS model. These weighting methods can be summarized into three kinds, objective weighting method (Chen et al., 2010; Zhang et al., 2007; Lu, 2003; Yang et al., 2008), subjective weighting method(Luo et al., 2011) and combination weighting method (Li et al., 2011; Zhang et al., 2009). Most papers concerning TOPSIS model have a preference for objective weighting method. W.-H. SU demonstrates that the excessive pursuit of objectivity about weighting methods ignores the subjective potency of human. The final weights may be unconscionable ( $\mathrm{Su}, 2011)$. L.-P. YU et al. argue that objective weighting methods cannot be entirely ensured. The future trend seems to conduct more combination weighting methods which combine subjective weighting methods and objective weighting methods (Yu et al., 2009). This paper proposes a combination weighting method which combines subjective weighting method 
(Analytic Hierarchy Process) and objective weighting method (Principal Component Analysis).

Euclid distance was replaced by Chi-square or Mahalanobis distance (Li et al., 2011; Wang et al., 2012) to overcome the possibility of alternatives closed to ideal point and nadir point concurrently. Grey system theory was also used to solve the problem (Chen et al., 2010; Sun et al., 2005). Some papers developed new evaluation methods (Liu et al., 1996). X.-Y. HUA et al. proposed a revised vertical projection method. In their paper, they defined a new distance: vertical projection distance and used vertical projection distance to replace Euclid distance (Hua et al., 2004). This method is simple and efficient, so in this paper, we use the vertical projection method for reference.

Finally, an improved TOPSIS model was used to evaluate NCAA basketball coaches after 1939 in the paper, whose feasibility and validity have been testified.

\section{TOPSIS Model}

TOPSIS (technique for order preference by similarity to solution) is a multiple criteria method to identify solutions from a finite set of alternatives. It first determines the ideal point and nadir point, then ranks all the alternatives according to their similarity to the ideal point.

The idea of TOPSIS can be expressed in the following steps (Olson, 2004).

1) Obtain performance data for $n$ alternatives over $k$ criteria. Raw data are usually standardized.

2) Develop a set of importance weights $\omega_{k}$ for each of the criteria.

3) Identify the ideal alternative (extreme performance on each criterion) and the nadir alternative (reverse extreme performance on each criterion).

4) Develop a distance measure over each criterion to both ideal point $\left(C^{*}\right)$ and nadir point $\left(C^{0}\right)$.

5) For each alternative, determine a ratio $f_{i}^{*}$ which represents the similarity to solution.

6) Rank order alternatives by maximzing the ratio $f_{i}^{*}$ in step 5 .

\section{Improved TOPSIS Model}

In the improved TOPSIS model, $\mathrm{R}$ cluster analysis was used to overcome correlations between criteria. A combination weighting method which combines subjective weighting method (Analytic Hierarchy Process) and objective weighting method (Principal Component Analysis) was presented. Vertical projection distance was used to replace Euclid distance which simplified the measurement of similarity to solution and the possibility of alternative closed to ideal point and nadir point concurrently was avoided.

\subsection{Normalization}

Y. P. LIAO et al. demonstrate that the vector normalization method was the best normalization method for TOPSIS model. It could deal with the general multi-attribute decision making (MADM) problems with various problem sizes, data ranges and attribute types effectively (Liao et al., 2012).

Use vector normalization method to standardized raw data.

$$
b_{i j}=\frac{a_{i j}}{\sqrt{\sum_{i=1}^{m} a_{i j}^{2}}}(i=1,2, \cdots, m ; j=1,2, \cdots, n)
$$

where $b_{i j}$ is a standardized measure converted from raw measure $a_{i j}$.

\subsection{Combination Weighting Method}

1)Subjective weighting method: Analytic Hierarchy Process

Some basic key steps involve Analytic Hierarchy Process are (Ovidya et al., 2006):

(a). State the problem and identify the criteria that influence the problem.

(b).Structure the problem in a hierarchy of different levels constituting goal, criteria, sub-criteria and alternatives.

(c).Compare each element in the corresponding level and calibrate them on the numerical scale.

(d).Perform calculations to find the maximum Eigen value, consistency index CI, consistency ratio CR, and normalized values for each criteria/alternative.

(e). If the maximum Eigen value, CI and CR are satisfactory, then decision is taken based on the normalized values; else the procedure is repeated till these values lie in a desired range. 
In practice, comparing one with another and constructing a judgment matrix according to the 1-9 scale may cause mistakes in the order of priorities. Y. -H. HOU et al. present that 1-9 scale is not fit for generating accurate weights, and exponential scale is the most accurate scale (Hou et al., 1995). Therefore, in this paper the exponential scale is used. Table 1 outlines the exponential scale.

Table 1. Exponential scale

\begin{tabular}{ll}
\hline Definition & Intensity of importance \\
\hline Equal importance & $9^{0}(1)$ \\
Moderate importance of one over another & $9^{(1 / 9)}(1.277)$ \\
Essential or strong importance & $9^{(3 / 9)}(2.080)$ \\
Very strong importance & $9^{(6 / 9)}(4.327)$ \\
Extreme importance & $9^{(9 / 9)}(9)$ \\
Intermediate values between the two adjacent judgements & $9^{(1 / K)}(K=0-9)$ \\
\hline
\end{tabular}

The random consistency index (R.I.) of exponential scale is as follows:

Table 2. Random consistency index (R.I.) of exponential scale (Wei, 2002)

\begin{tabular}{cccccccc}
\hline$n$ & 3 & 4 & 5 & 6 & 7 & 8 & 9 \\
\hline RI & 0.32 & 0.53 & 0.66 & 0.75 & 0.81 & 0.86 & 0.89 \\
\hline
\end{tabular}

2) Objective weighting method: Principal Component Analysis (Wu et al., 2009)

PCA (Principal Component Analysis) is the simplest of the true eigenvector-based multivariate analyses. Often, its operation can be considered as revealing the internal structure of the data in a way that best explains the variance in the data. So, this paper chooses PCA as an objective weighting method. The steps of PCA are as follows:

(a). Standardize raw data

There are $n$ criteria $\left(x_{1}, x_{2}, \cdots, x_{n}\right)$ and $m$ alternatives. The value of number $i$ alternative in number $j$ criterion is $a_{i j}, a_{i j}$ is standardized to be $\tilde{a}_{i j}$.

$$
\tilde{a}_{i j}=\frac{a_{i j}-\mu_{i j}}{s_{j}} \quad(i=1,2, \cdots, m ; j=1,2, \cdots, n),
$$

where

$$
\begin{gathered}
\mu_{i j}=\frac{1}{n} \sum_{i=1}^{m} a_{i j} \\
s_{j}=\sqrt{\frac{1}{n} \sum_{i=1}^{m}\left(a_{i j}-\mu_{j}\right)^{2}} \quad(j=1,2, \cdots m)
\end{gathered}
$$

(b). Obtain the correlation coefficient matrix $R . R=\left(r_{i j}\right)_{n \times n}$. Then

$$
r_{i j}=\frac{\sum_{k=1}^{n} \tilde{a}_{k i} \cdot \tilde{a}_{k j}}{n-1} \quad(i, j=1,2, \cdots, n)
$$

Where $r_{i i}=1, r_{i j}=r_{j i}, \quad r_{i j}$ is the correlation coefficient of criteria $i$ and $j$.

(c). Calculate the eigenvalue and eigenvector. Calculate the eigenvalue of correlation coefficient matrix $R$, $\lambda_{1} \geq \lambda_{2} \geq \cdots \geq \lambda_{n} \geq 0$. Then calculate the corresponding eigenvector $u_{1}, u_{2}, \cdots, u_{n}$, where $u_{j}=\left[u_{1 j}, u_{2 j}, \cdots, u_{n j}\right]^{T}$. The final $n$ principal components are as follows:

$$
\begin{gathered}
y_{1}=u_{11} \tilde{x}_{1}+u_{21} \tilde{x}_{2}+\cdots+u_{n 1} \tilde{x}_{n} \\
y_{2}=u_{12} \tilde{x}_{1}+u_{22} \tilde{x}_{2}+\cdots+u_{n 2} \tilde{x}_{n} \\
\vdots \\
y_{n}=u_{1 n} \tilde{x}_{1}+u_{2 n} \tilde{x}_{2}+\cdots+u_{n n} \tilde{x}_{n}
\end{gathered}
$$

(d). Calculate the contribution ratio $\left(b_{j}\right)$ of eigenvalue $\lambda_{j}(j=1,2, \cdots, n)$. 


$$
b_{j}=\frac{\lambda_{j}}{\sum_{k=1}^{m} \lambda_{k}} \quad j=1,2, \cdots, n
$$

(e). Calculate the weights of the $n$ criteria.

$$
\begin{gathered}
Y=\sum_{j=1}^{n} b_{j} y_{j} \\
Y=c_{1} \widetilde{x}_{1}+c_{2}{\widetilde{x_{2}}}+\cdots+c_{n} \widetilde{x_{n}}
\end{gathered}
$$

Where $\tilde{x}_{1}, \tilde{x}_{2} \cdots \tilde{x}_{n}$ are the criteria after standardization.

The weight of criterion $x_{j}$ is $\omega_{j}$.

$$
\omega_{j}=\frac{c_{j}}{\sum_{j=1}^{n} c_{j}} \quad(j=1,2, \cdots, n)
$$

Where $c_{j}$ is the coefficient of $\tilde{x}_{j}$ in formula (7).

For a criterion, the average value of its weights obtained from subjective weighting method and objective weighting method is its combination weight.

\subsection{Weighted Matrix}

$C=\left(c_{i j}\right)_{m \times n}$ is the weighted matrix,

$$
c_{i j}=\omega_{j} \cdot b_{i j}, i=1,2, \cdots, m ; j=1,2, \cdots, n .
$$

Where $\omega=\left[\omega_{1}, \omega_{2}, \cdots, \omega_{n}\right]^{T}$ is the final weight vector and $b_{i j}$ is the standardized measure converted from raw measure $a_{i j}$.

\subsection{Determining Ideal Point and Nadir Point}

Determine ideal point $C^{*}$ and nadir point $C^{0} . c_{j}^{*}$ is the value of $C^{*}$ in number $j$ criterion. $c_{j}^{0}$ is the value of $C^{0}$ in number $j$ criterion.

ideal point:

$$
\begin{gathered}
c_{j}^{*}=\max c_{i j}, j(j=1,2, \cdots, n) \quad \text { (benefit attribute) } \\
c_{j}^{*}=\min c_{i j}, j(j=1,2, \cdots, n) \quad \text { (cost attribute) } .
\end{gathered}
$$

nadir point:

$$
\begin{gathered}
c_{j}^{0}=\max c_{i j}, j(j=1,2, \cdots, n) \quad(\text { cost attribute }) \\
c_{j}^{0}=\min c_{i j}, j(j=1,2, \cdots, n) \quad \text { (benefit attribute). }
\end{gathered}
$$

Where $c_{j}^{*}$ is the value of number $j$ attribute of ideal point $C^{*}, c_{j}^{0}$ is the value of number $j$ attribute of nadir point $C^{0}$.

\subsection{Measuring Similarity by Vertical Projection Distance}

3) Traditional method: measuring similarity to solution by Euclid distance

Similarity to solution is alternative's similarity to ideal point.

Calculate the distances of alternatives between ideal point and nadir point. The distance between alternative and ideal point $C^{*}$ is $s_{i}^{*}$, 


$$
s_{i}^{*}=\sqrt{\sum_{j=1}^{n}\left(c_{i j}-c_{j}^{*}\right)^{2}} \quad(i=1,2, \cdots, m)
$$

The distance between alternative and nadir point $C^{0}$ is $s_{i}^{0}$ :

$$
s_{i}^{0}=\sqrt{\sum_{j=1}^{n}\left(c_{i j}-c_{j}^{0}\right)^{2}} \quad(i=1,2, \cdots, m)
$$

Calculate the similarity to solution $f_{i}^{*}$ :

$$
f_{i}^{*}=\frac{s_{i}^{0}}{s_{i}^{0}+s_{i}^{*}} \quad(i=1,2, \cdots, m)
$$

4) New method: measure similarity to solution by vertical projection distance (Hua et al., 2004)

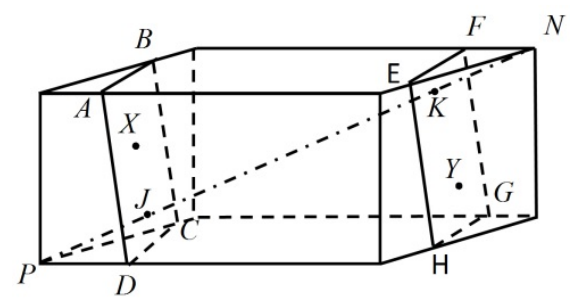

Figure 1. Presentation of vertical projection distance

In Figure 1, $P$ and $N$ represent ideal point and nadir point. The vertical projection distance from $X$ to $Y$ represents the distance between two planes whose normal vector is straight line $P N$. One of them crosses $X$ and the other crosses $Y$.

Vertical projection distance reflects the similarity to solution, X. -Y. HUA et al. demonstrate that when the vertical projection distance between an alternative and the ideal point is shorter, the vertical projection distance between the alternative and the nadir point will be longer (Hua et al., 2004).

Some key and basic steps involved vertical projection method are as follows:

In order to simplify calculation, translate the origin of coordinate to the ideal point firstly. The matrix after translation is $T=\left(t_{i j}\right)_{m \times n}$, where $t_{i j}=c_{i j}-c_{j}^{*} \quad(i=1,2, \cdots, m \quad j=1,2, \cdots, n)$.

After translation, the ideal point is $\{0,0, \cdots, 0\}$, the nadir point is $\mathrm{H}^{-}=\left\{H_{j}^{-}=\left(c_{j}^{0}-c_{j}^{*}\right) j=1,2, \cdots, n\right\}$.

X. -Y. HUA et al. demonstrate that the vertical projection distance between a alternative and the ideal point can be expressed as follows:

$$
P_{i}=\sum_{j=1}^{n} H_{j}^{-} t_{i j}
$$

The smaller the value of $P_{i}$ is, the better the alternative will be (Hua et al., 2004). The proofs of the conclusions discussed before can be found in paper by Hua et al.

\section{Improved TOPSIS Model's Application in NCAA Basketball Coach Evaluation}

\subsection{Screening Criteria}

The National Collegiate Athletic Association (NCAA) is a non-profit association that organizes the athletic programs of many colleges and universities in the United States and Canada. NCAA basketball is one of the most popular programs. This paper evaluated all the NCAA basketball coaches after 1939. Primarily, according to the related website's statistical data, 10 criteria were confirmed. 
Table 3. 10 Criteria

\begin{tabular}{cll}
\hline \multicolumn{3}{c}{ NumberAbbreviation } \\
\hline 1 & Yrs & years \\
2 & G & games \\
3 & W & wins \\
4 & L & losses \\
5 & W-L\% & Win-Loss percentage \\
6 & CREG & Number of regular season conference champions won \\
7 & CTRN & Number of conference tournament championships won \\
8 & NCAA & Number of NCAA Tournament appearances \\
9 & FF & Number of NCAA Final Four appearances \\
10 & NC & Number of NCAA Tournament championships won \\
\hline
\end{tabular}

There are obvious correlations among the 10 criteria. R cluster analysis was used to classify the 10 criteria. Then we selected the most representative criterion in each group. The cluster tree is shown in Figure 2.

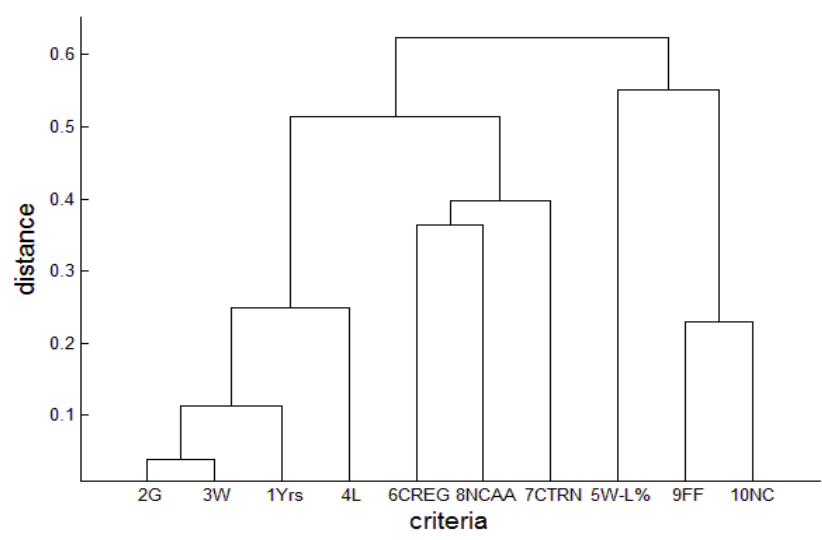

Figure 2. Cluster tree

According to Figure 2, 4 groups are created. First group: 1, 2, 3, 4; second group: 6, 7, 8; third group: 5; fourth group: $9,10$.

According to intuitive judgment, we can see that there is high correlation among 1, 2, 3, 4. 2 games, 3 wins, 4 losses will increase as 1 years increase. According to NCAA basketball competition system, 6, 7, 8 are of high correlation and there is also high correlation between 9,10 . So the result of R cluster analysis is reasonable.

In the first, second and third group, we selected criteria 1, 7, 5. In the fourth group, a new criterion named FFNC is created. 9 and 10 are added according to the proportion of 1:4.

Finally $x_{1}, x_{2}, x_{3}, x_{4}$ are used to represent the four criteria: Yrs, W-L\%, CTRN and FFNC.

\subsection{Obtaining Weights about Criteria}

\section{1) Analytic Hierarchy Process}

The hierarchy for obtaining the weights of criteria is shown below in Figure 3.

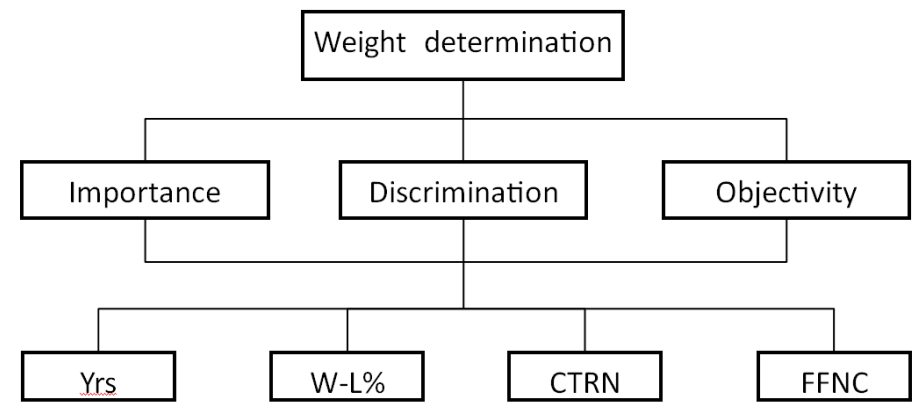

Figure 3. The hierarchy 
We have the following for the matrix of pairwise comparisons of the criteria with respect to the overall focus.

C.R. $=0.0035$

\begin{tabular}{|c|c|c|c|c|}
\hline Focus & Importance I & Discrimination & nObjectivity & $\mathrm{Wt}$ \\
\hline Importance & 1 & $9^{4 / 9}(2.63)$ & $9^{6 / 9}(4.36)$ & 0.6215 \\
\hline Discrimination & $9^{-(4 / 9)}(0.38)$ & 1 & $9^{2 / 9}(1.62)$ & 0.2345 \\
\hline Objectivity & $9^{-(6 / 9)}(0.23)$ & $9^{-(2 / 9)}(0.62)$ & 1 & 0.1440 \\
\hline
\end{tabular}

We obtain the vector of relative weights:

(Importance, Discrimination, Objectivity $)=(0.6215,0.2345,0.1440)$.

\begin{tabular}{|c|c|c|c|c|c|}
\hline Importanc & Yrs & W-L\% & CTRN & FFNC & $\mathrm{Wt}$ \\
\hline Yrs & 1 & $9^{-(3 / 9)}(0.48)$ & $9^{-(5 / 9)}(0.30)$ & $9^{-1}(0.11)$ & 0.0661 \\
\hline W-L \% & $9^{(3 / 9)}(2.08)$ & & $(0.89)$ & $9^{-(6 / 9)}(0.2$ & 0.1521 \\
\hline CTRN & $9^{(5 / 9)}(3.39)$ & $9^{(0.5 / 9)}(1.13) 1$ & & $9^{-(3 / 9)}(0.48$ & 0.2192 \\
\hline FFNC & 9 & $9^{(6 / 9)}(4.33)$ & $9^{(3 / 9)}(2.08)$ & 1 & 0.5626 \\
\hline
\end{tabular}

C.R. $=0.0235$

the local derived weight scales for the criteria Importance, Discrimination and Objectivity are as follows:

a. $($ Yrs, W-L\%, CTRN, FFNC $)=(0.0661,0.15210 .21920 .5626)$. C.R. $=0.0235$

b. (Yrs, W-L\%, CTRN, FFNC $)=(0.09650 .17960 .1293$ 0.5946).C.R. $=-0.0873$

c. $($ Yrs, W-L\%, CTRN, FFNC) $=(0.09060 .30310 .30310 .3031) . C . R .=0.0080$

All the consistency ratios do not exceed 0.10 . To synthesize the overall weight scale we multiply as follows:

$$
\left(\begin{array}{lll}
0.0661 & 0.0965 & 0.0906 \\
0.1521 & 0.1796 & 0.3031 \\
0.2192 & 0.1293 & 0.3031 \\
0.5626 & 0.5946 & 0.3031
\end{array}\right)\left(\begin{array}{l}
0.6215 \\
0.2345 \\
0.1440
\end{array}\right)=\left(\begin{array}{l}
0.0768 \\
0.1803 \\
0.2102 \\
0.5327
\end{array}\right)
$$

The final weights of the four criteria $x_{1}, x_{2}, x_{3}, x_{4}$ by AHP are (0.0768 0.18030 .21020 .5327$)$.

2) Principal Component Analysis

4 principal components are obtained by PCA, $y_{1}, y_{2}, y_{3}, y_{4}$. Their structures are as follows:

Table 4. Structures of the 4 principal components

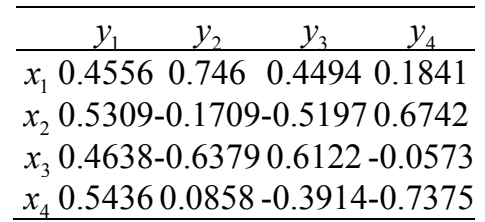

The contribution ratio of $y_{1}, y_{2}, y_{3}, y_{4}$ are: $0.5077,0.1910,0.1672,0.1341$.

According to foregoing information and formula (6), weights of $x_{1}, x_{2}, x_{3}, x_{4}$ can be obtained.

$$
Y=0.4736 x_{1}+0.2404 x_{2}+0.2083 x_{3}+0.1280 x_{4}
$$

After normalization processing, the weights of $x_{1}, x_{2}, x_{3}, x_{4}$ by PCA are $0.4509,0.2289,0.1893,0.1219$.

3) Combination weights

The final weights of $x_{1}, x_{2}, x_{3}, x_{4}$ are as follows: 
Table 5. Final weights

\begin{tabular}{|c|c|c|c|c|}
\hline & $x_{1}$ & $x_{2}$ & $x_{3}$ & $x_{4}$ \\
\hline PCA & \multicolumn{4}{|c|}{0.45090 .22890 .18930 .1219} \\
\hline AHP & \multicolumn{4}{|c|}{0.07680 .18030 .21020 .5327} \\
\hline \multicolumn{5}{|c|}{ Combination weights 0.26390 .20460 .19980 .3273} \\
\hline
\end{tabular}

\subsection{Model Solution}

Apply the combination weights to the improved TOPSIS model. The top 32 coaches are as follows:

Table 6. The top 32 coaches

\begin{tabular}{crrr}
\hline ranking & \multicolumn{1}{c}{ coaches } & ranking & \multicolumn{1}{c}{ coaches } \\
\hline 1 & John Wooden & 17 & Jim Boeheim \\
2 & Mike Krzyzewski & 18 & John Thompson \\
3 & Adolph Rupp & 19 & Nolan Richardson \\
4 & Dean Smith & 20 & Fred Taylor \\
5 & Jim Calhoun & 21 & Steve Fisher \\
6 & Bob Knight & 22 & Phog Allen \\
7 & Rick Pitino & 23 & Bill Self \\
8 & Roy Williams & 24 & Joe B. Hall \\
9 & Denny Crum & 25 & Frank McGuire \\
10 & Hank Iba & 26 & Gary Williams \\
11 & Billy Donovan & 27 & Nat Holman \\
12 & Tom Izzo & 28 & Doggie Julian \\
13 & John Calipari & 29 & Don Haskins \\
14 & Branch McCracken & 30 & Tubby Smith \\
15 & Lute Olson & 31 & Kenneth Loeffler \\
16 & Jerry Tarkanian & 32 & Pete Newell \\
\hline
\end{tabular}

American sports media Bleacher Report selected the 10 most greatest college basketball coaches. They are as follows.

Table 7. Bleacher Report's ranking

\begin{tabular}{crr}
\hline Bleacher Report's ranking & \multicolumn{1}{c}{ coaches } & This paper's ranking \\
\hline 1 & John Wooden & 1 \\
2 & Bob Knight & 6 \\
3 & Mike Krzyzewski & 2 \\
4 & Adolph Rupp & 3 \\
5 & Dean Smith & 4 \\
6 & Jim Calhoun & 5 \\
7 & Jim Boeheim & 17 \\
8 & Lute Olson & 15 \\
9 & Eddie Sutton & $>32$ \\
10 & Jim Phelan & $>32$ \\
\hline
\end{tabular}

According to table 7, the top 6 coaches are very similar between Bleacher Report's and this paper's ranking.

ESPN (Entertainment and Sports Programming Network) selected 8 greatest college basketball coaches without ranking. They are as follows:

Table 8. ESPN's ranking

\begin{tabular}{rrrr}
\hline Before 1980s & This paper's ranking & After 1980s & This paper's ranking \\
\hline John Wooden & 1 & Dean Smith & 4 \\
Hank Iba & 10 & John Thompson & 18 \\
Adolph Rupp & 3 & Bob Knight & 6 \\
Pete Newell & 32 & Mike Krzyzewski & 2 \\
\hline
\end{tabular}


According to table 8 , the 8 greatest coaches are all in this paper's ranking.

This paper only considers some quantifiable factors, but Bleacher Report and ESPN considered some factors that cannot be quantified. So there are some differences about the ranking.

The improved TOPSIS model proposed in this paper has three advantages:

1) This paper proposes a new method on evaluation index system based on R cluster analysis, which overcome the correlations between criteria.

2) This paper presents a combination weighting method which combines subjective weighting method (Analytic Hierarchy Process) and objective weighting method (Principal Component Analysis). This weighting method considered subjective potency of human and the variance in the data at the same time. This method makes final weight more reasonable.

3) This paper introduces vertical projection method for reference. The possibility of alternative closed to ideal point and nadir point concurrently was avoided. The measurement of similarity to solution was simplified.

\section{Conclusion}

The main purpose of this paper is to propose a more systematic TOPSIS model, which is easy to apply in different fields. This paper uses its application on the evaluation of NCAA basketball coaches as an example. This improved TOPSIS model can also be easily applied to other fields, including the evaluation of other kinds of coaches, employee performance review and supplier selection, etc.

In the improved TOPSIS model, the correlations between criteria were overcome by a new method on the evaluation index system based on $\mathrm{R}$ cluster analysis. The paper also proposes a combination weighting method which considered both subjective potency of human and the variance in the data. The possibility of alternative closed to ideal point and nadir point concurrently was avoided by vertical projection method and the measurement of the similarity to solution was simplified. The feasibility and validity of this improved model are testified by the evaluation of NCAA basketball coaches, however, the evaluation was based on 10 criteria, which are quantifiable factors which can be collected easily, and may not be comprehensive enough. The final results will be more accurate if more criteria are obtained and the subjective factors are quantified.

\section{Acknowledgements}

The authors acknowledge the financial support of this research by the Key Laboratory of Product Packaging and Logistics of Guangdong Higher Education Institutes, the Fundamental Research Funds for the Central Universities, the project of the Natural Science Foundation of Guangdong Province (No.S2012010008773), and the projects of Zhuhai Science, Technology, Industry, Trade and Information Technology Bureau (No.2011B050102013 \& 2012D0501990033).

\section{References}

Bleacher, Report. (2012). 10 Greatest Coaches in NCAA Basketball History. Retrieved from http://bleacherreport.com/articles/1341064-10-greatest-coaches-in-ncaa-basketball-history

Chen, H., \& Zhang, Q. S. (2010) An improved Weighted TOPSIS Method and Its Application Based on Grey Relational Analysis. Journal of Fuzhou University, 6, 40-44.

ESPN Men's Basketball (2006). College hoops Rushmores: ESPN-era coaches. Retrieved from http://insider.espn.go.com/ncb/insider/columns/story?id=2422347

Hou, Y. H., \& Shen, D. J. (1995). Index Number Scale and Comparison with Other Scales. Systems Engineering Theory \& Practice, 10, 43-46.

Hua, X. Y., \& Tan, J. X. (2004). Revised TOPSIS Method Based on Vertical Projection Distance-Vertical Projection Method. Systems Engineering-theory \& Practice, 1, 114-119.

Li, X. F., Liu, Z. X., \& Peng, Q. E. (2011). Improved Algorithm of TOPSIS Model and Its Application in River Health Assessment. Journal of Sichuan University, 43(2), 14-21.

Liao, Y. P., Liu L., \& Xing, C. (2012). Investigation of Different Normalization Methods for TOPSIS. Transactions of Beijing Institute of Technology, 32(5), 871-875, 880.

Liu, S. L., \& Qiu, W. H. (1996). The TOPSIS Angle Measure Evaluation Method for MADM. Systems Engineering Theory \& Practice, 7, 12-16.

Lu, F. Y. (2003). An improved TOPSIS method. Statistics and Decision, 3, 78. 
Luo, X. X., \& Peng, S. H. (2011). Research on the Vendor Evaluation and Selection Based on AHP and TOPSIS in Green Supply Chain. Soft Science, 25(2), 53-56.

Olson, D. L. (2004). Comparison of Weights in TOPSIS Models. Mathematical and Computer Modelling, 40, 721-727. http://dx.doi.org/10.1016/j.mcm.2004.10.003

Omkarprasad, S. V., \& Sushil, K. (2006). Analytic hierarchy process: An overview of applications. European Journal of Operational Research, 169, 1-29. http://dx.doi.org/10.1016/j.ejor.2004.04.028

SR/COLLEGE BASKETBALL. Coaches. Retrieved from http:/www.sports-reference.com/cbb/coaches/

$\mathrm{Su}, \mathrm{W} . \mathrm{H}$. (2000). Multiple-Objective-Comprehensive-Evaluation theory research and methodology. Xiamen: Xiamen University.

Sun, X. D., Jiao, Y., \& Hu, J. S. (2005). Research on Decision-Making Method Based on Gray Correlation Degree and TOPSIS. Chinese Journal of Management Science, 13(4), 63-68.

Wang, X. J., \& Wang, L. (2012). Applications of TOPSIS improved based on Mahalanobis distance in supplier selection. Control and Decision, 27(10), 1566-1570.

Wei, Z. Z. (2012). Using Computer Algebra to Calculate the Linear Oscillator. Journal of Guangxi Teachers College, 19(2), 26-29.

Wu, T. F., Deng, H. Q., \& Wu, R. T. (2009). Calculation of the Force Structure's Weight Based on PCA. Microcomputer Information, 25(6-1), 264-265.

Yang, Z. Z., et al. (2008). A Study on the Method for Supplier Selection Based on Principal Component Analysis and TOPSIS. Journal of Information, 11, 7-10.

Yu, L. P., Pan, Y. T., \& Wu, Y. S. (2009). Comparing objective weighting with subjective weighting in Sci-tech education institute assessment. Science Research Management, 30(4), 154-161.

Zhang, J., \& Lian, C. (2009). Application of TOPSIS Model Based on Gray Correlation Coefficient Matrix in the Evaluation of Water Environmental Quality. Journal of Sichuan University, 41(4), 97-101.

Zhang, X. Q., Liang, C., \& Liu, H. Q. (2007). Application of improved TOPSIS method based on coefficient of entropy to comprehensive evaluating water quality. Journal of Harbin Institute of Technology, 39(10), $1670-1672$.

\section{Copyrights}

Copyright for this article is retained by the author(s), with first publication rights granted to the journal.

This is an open-access article distributed under the terms and conditions of the Creative Commons Attribution license (http://creativecommons.org/licenses/by/3.0/). 